\title{
Reliability Evaluation and In-Orbit Residual Life Prediction for Satellite Lithium-Ion Batteries
}

\author{
Bin Yu $\mathbb{D}^{1,2}$ Tao Zhang, ${ }^{1}$ Tianyu Liu $\mathbb{D}^{1,3}$ and Lei Yao ${ }^{1,2}$ \\ ${ }^{1}$ College of System Engineering, National University of Defense Technology, Changsha, China \\ ${ }^{2}$ China Aerospace and Technology Corporation, Beijing, China \\ ${ }^{3}$ Troop 78092 of PLA, China \\ Correspondence should be addressed to Bin Yu; yubin_nudt@163.com
}

Received 24 July 2018; Accepted 22 November 2018; Published 6 December 2018

Academic Editor: Alessandro Naddeo

Copyright (c) 2018 Bin Yu et al. This is an open access article distributed under the Creative Commons Attribution License, which permits unrestricted use, distribution, and reproduction in any medium, provided the original work is properly cited.

\begin{abstract}
As a new type of secondary battery, lithium-ion battery is widely used in the aerospace industry with the advantages of long lifetime, high energy density and low pollution, etc. In this paper, we focus on the problem of offline and online life prediction for satellite lithium-ion batteries. Firstly, based on the NASA laboratory battery dataset, a Wiener process with time-scale transformation is used to capture battery capacity fading, and then the battery reliability point and interval estimation equation are derived, respectively. Secondly, by analyzing the charge and discharge profiles of the batteries in orbit environments, an accurate capacity prediction model is proposed based on the partial charging curves. Finally, the Bayesian framework is used to perform capacity degradation model online updating, and the analytical expression of residual life distribution is derived to achieve RL prediction for in-orbit satellite lithium-ion batteries.
\end{abstract}

\section{Introduction}

As a promising secondary battery, lithium-ion battery develops rapidly in recent ten years and has been widely used in digital electronics (mobile phone, camera, laptop, tablet PC, etc.), electric vehicles, and grid energy storage systems [1]. Researches show that lithium-ion battery is significantly superior to other traditional batteries in energy density, cycle life, self-discharge rate, memory effect, and being environment friendly [2]. These advantages make lithium-ion battery gain continuous attentions from aerospace engineers. Since the birth of satellite, its energy storage system (ESS) has developed three generations. The first and second generations of ESS use cadmium-nickel and nickel-hydrogen batteries, respectively, which weigh $40 \%$ and $20 \%$ of the total weight of a satellite. By comparison, the third-generation ESS adopts lithium-ion batteries as energy intermediate storage, whose energy density can reach 2 to 4 times that of old generation batteries. Some microsatellites, for example, the STRV-1d launched in 2000, the PROBA launched in 2001, and the ST-5 launched in 2006, firstly used lithium-ion batteries to power the load. With the successful application of small satellites, lithium-ion batteries have gradually spread to some highorbit satellites, such as the W3A satellite launched by the European Space Agency in 2004, the Amazonas series of satellites launched by Hispamar, and the Skynet 5 series of satellites owned by the UK Ministry of Defense.

Compared with the applications on the ground, aerospace products put forward higher requirement in reliability and safety. Generally, failure of batteries will affect the normal operation of satellite loadings and even lead to catastrophic accidents. For example, batteries accidental failure resulted in the halting problem of the Mars Global Surveyor in 2006 [3] and Boeing 787 in 2013 [4]. Therefore, the techniques of life prediction play an increasingly significant role in satellite lithium-ion batteries.

According to a new product's development stage, life prediction can be divided into offline prediction and online prediction. Offline prediction mainly occurs in the stage of battery design and manufacture, which focuses on the problem of reliability evaluation for the population of a given type battery. By comparison, online prediction concerns more about the issue of residual life (RL) prediction for an individual battery in operation environments $[5,6]$. Here, 
the RL of a lithium-ion battery refers to the length of cycle time from present to the end of life (EOL) in orbit. For the long lifetime and highly reliable products, researchers often perform life prediction based on the degradation data instead of failure time data, which are quite scarce in practical applications.

In the literature, the capacity induced by the chargedischarge cycle is a suitable feature to characterize battery degeneration. Numerous studies of lithium-ion battery life prediction are transformed to the issue of capacity degradation modeling and first hitting time assessment. Jin et al. [7] presented a framework of lithium-ion battery capacity degradation assessment and RL prediction based on the techniques of Wiener process and particle filtering. Xing et al. [8] used an ensemble regression model to capture lithium-ion battery capacity fading and then realized the remaining useful performance prediction. Saha et al. [9] studied the characteristics of lithium-ion battery capacity variation over time, and an empirical degradation model under the framework of Bayesian is utilized to perform RL prediction. Nuhic et al. [10] provided a support vector machine (SVM) based approach on lithium-ion battery state of health $(\mathrm{SOH})$ forecasting and RL reliable prediction.

One thing these researches all have in common is that the capacity is used as the health indicator for data modeling and inferring. Conventionally, the capacity of secondary battery can be accurately measured in a complete chargedischarge cycle, which can be performed conveniently in the laboratory. However, for the online case (like the inorbit environments), it is very difficult to perform capacity measurement and monitoring [11]. The reasons mainly are twofold: (a) A complete charge/discharge cycle consumes several hours and may affect the normal operation of satellite loadings. Although high current rate can shorten the testing time, it will lead to serious capacity underestimation and battery aging acceleration; (b) deep discharge will cause permanent damage to a lithium-ion battery and shorten its operation time. Some studies attempt to use other online available features, such as impedance, temperature, or parameters in the current/voltage curves as battery health indicator, but the prediction effects are less effective than capacity. For example, Saha et al. [12] proposed a battery health monitoring and prognostics approach based on two types of impedance. However, the features must be extracted by the electrochemical impedance spectroscopy (EIS) equipment, which is space consuming and not suitable installed in a satellite. Liu et al. [11] and Feng et al. [13] extracted two features, the time-to-voltage-saturation (TVS) and the timeto-current-saturation (TCS), from the charging curves to perform residual life prediction. But this method needs the full charging curve in each cycle which is also difficult to acquire in orbits.

In a word, capacity is still the most reliable feature in lithium-ion battery life prediction. To perform in-orbit health prognostics, an accurate capacity prediction model should be developed firstly. In this paper, a framework of online and offline life prediction for satellite lithium-ion batteries was studied. Considering the characteristics of randomness and nonlinearity in capacity fading, a Wiener stochastic process with time-transformation was used to model battery performance degradation with offline data. With a specified threshold, both the point estimate and confidence intervals (CIs) of the reliability function and life indices were derived. In online stage, we firstly analyzed the charge/discharge profiles of a battery in orbit and then developed an empirical stepwise regression model for capacity accurate prediction. With the predicted capacity, a Bayesian method is developed subsequently to realize model parameter updating and RL prediction, which can ensure the prediction accuracy considering battery item-to-item heterogeneity.

The remainder of this paper is organized as follows. Section 2 gives the methods of offline capacity fading modeling and reliability evaluation. Section 3 proposes the approach of online capacity prediction for in-orbit satellite batteries. In Section 4, a Bayesian based in-orbit residual life prediction model is established. Finally, Section 5 concludes this paper.

\section{Offline Capacity Fading Modeling and Reliability Evaluation}

2.1. Experimental Data and Failure Mechanisms Analysis. The dataset in this paper is supplied by the Prognostics Center of Excellence (PCoE) in NASA Ames Research Center [14]. To study the performance degradation and health prognostic methods of lithium-ion batteries for satellite usage, four 18650 cylindrical commercial lithium-ion cells, indexed as B0005, B0006, B0007, and B0018, were tested in this center. The rated capacity, charge cut-off voltage, and discharge cut-off voltage of these batteries are $2 \mathrm{Ah}, 4.2 \mathrm{~V}$, and $2.7 \mathrm{~V}$, respectively. Although these samples are selected from the same population, the randomness in materials and manufactures makes them show some heterogeneity in performance and lifetime.

A standard constant current/voltage (CC-CV) charge and constant current (CC) discharge regime is adopted in the test. The testing temperature is controlled at 25 Celsius by a temperature chamber to simulate in-orbit conditions. The steps of CC-CV and CC regime in one cycle are as follows:

(1) Charge with the constant current of $1.5 \mathrm{~A}$ until the voltage reaches $4.2 \mathrm{~V}$;

(2) Charge with the constant voltage of until the current declines to $0.02 \mathrm{~A}$;

(3) Discharge with the constant current of $2 \mathrm{~A}$ until the voltage declines to $2.7 \mathrm{~V}$;

(4) Stand by for 350 seconds.

The tests are terminated after 166 cycles on B0005, B0006, B0007, and 131 cycles on B0018.

Figures 1 and 2 illustrate the variations of voltage and current curves of B0005 as cycle number increases. As the testing proceeds, battery performance degrades gradually. In the charge phase, the CC charging time decreases while the $\mathrm{CV}$ charging time increases. In other words, the voltage of a used battery rises faster than a new one in the CC charging, and the current of a new battery drops faster than an old one in the CV charging. In the CC discharging phase, battery aging leads to an apparent decrease of discharge duration 


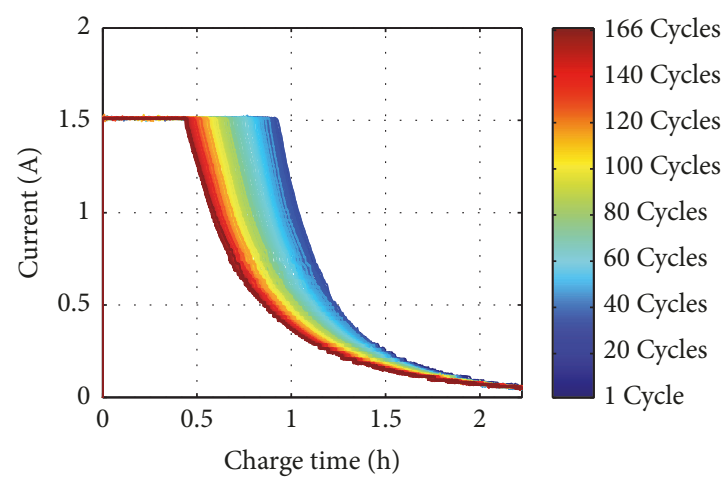

(a) Charge voltage

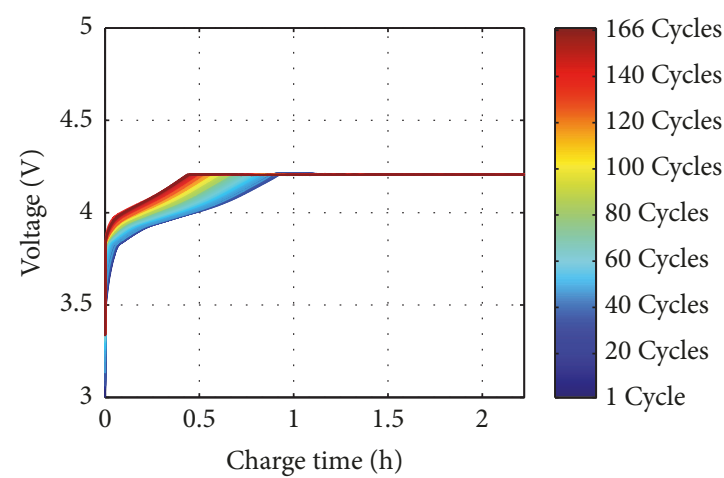

(b) Charge current

FIGURE 1: Variations of battery voltage and current curves in the CC-CV charging.

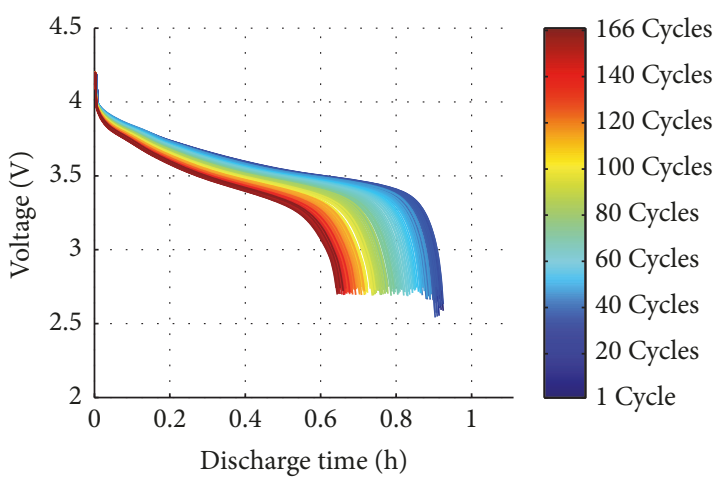

FIGURE 2: Variation of battery voltage curve in the CC discharging.

under constant current, indicating that a fully charged battery supplies less energy for the loadings.

Theoretically, there are various feature parameters reflecting a battery's health status. In the literature, capacity is most frequently used to determine lithium-ion batteries' endof-life (EOL) and further perform degradation modeling and lifetime prediction. In laboratory conditions, battery capacity can be easily acquired by the Ampere-hour integral method. By comparison, other features (such as impedance, temperature, or voltage platform) are quite susceptible to the external factors (like loading current, DOD, SOC, etc.), and measuring them accurately is a relatively intractable work. As Figure 3 shows, a battery's capacity can be measured by fully charging it and integrating the charging current $\left(C_{1}\right)$, or fully discharging it and integrating the discharge current $\left(C_{2}\right)$. Here, fully charging (or discharging) corresponds to the variation of state-of-charge (SOC) from 0 to $100 \%$ (or 100\% to 0 ). Since the two ways yield close results in each cycle, for the rest of this paper no differentiation between $C_{1}$ and $C_{2}$ is made.

The capacity degradation during the test for the four batteries is shown in Figure 4. The y-axis "capacity loss" is scaled by dividing the degradation increment by the rated value (2Ah). Researches show that the growth of the solid electrolyte interface (SEI) mainly contributes to the capacity fading of lithium-ion batteries. During charge/discharge

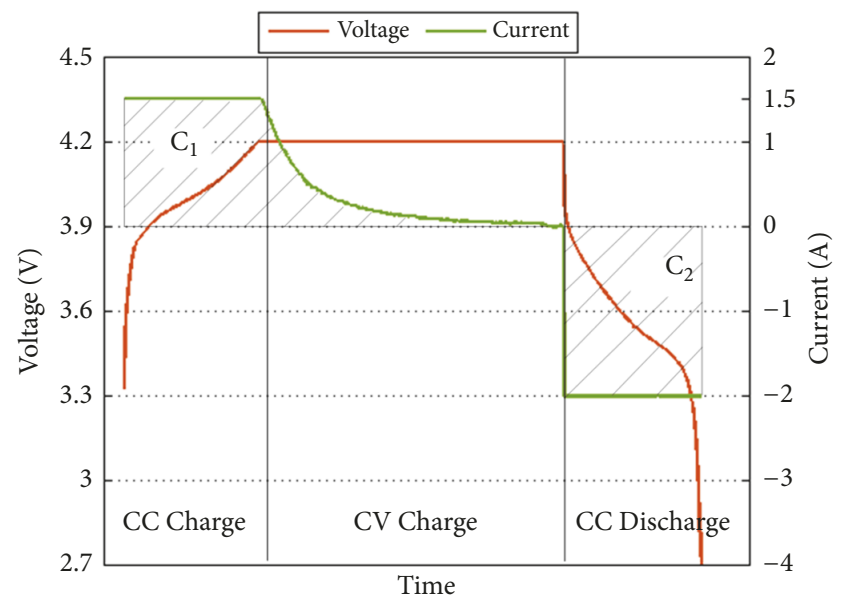

FIGURE 3: Voltage and current curve in a cycle.

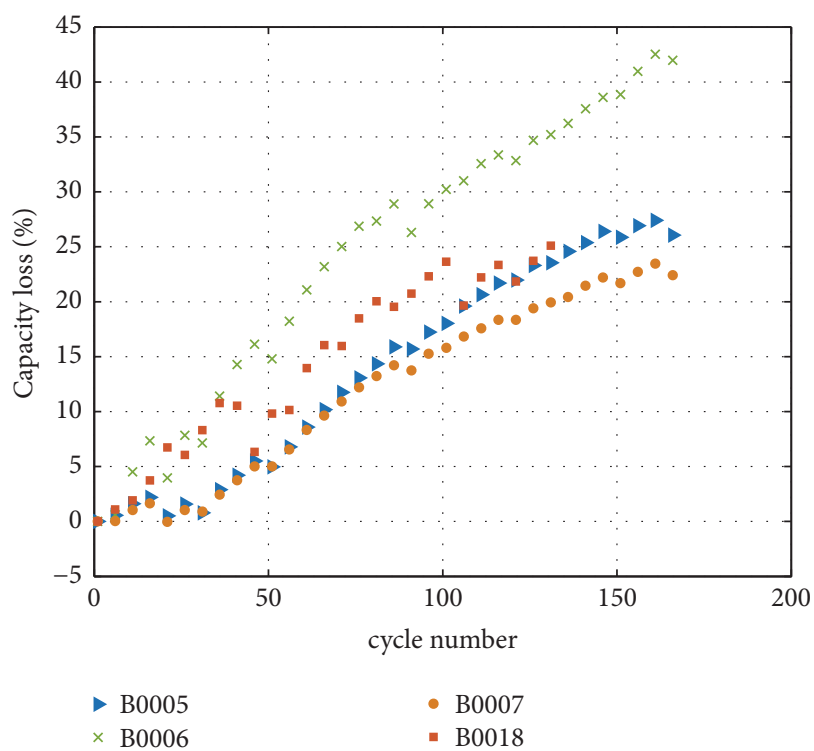

FIgURE 4: Capacity loss vs cycle numbers. 
cycle, the current generates diffusion induced stress inside a battery and triggers cracks on the graphite particles. Consequently, SEI grows at the interface of cathode and electrolyte, which consumes $\mathrm{Li}^{+}$and active materials at the anode and cathode. Besides SEI growth, some other unexpected side reactions, like the oxidation of anode, lithium corrosion on cathode, and electrolyte decomposition, also consume active $\mathrm{Li}^{+}$and result in capacity loss. According to the first principle, the rate of side reactions decreases as aging progresses. As a result, the speed of capacity fading appears first quick back slow trend. Considering the engineering experience in satellite design, a battery is considered to fail when its capacity loss reaches $30 \%$.

2.2. Capacity Degradation Modeling. Capacity fading is stochastic in nature due to the inherent inconsistency in battery materials, manufactures, and environment changes. As a famous non-monotone stochastic process, the Wiener process is quite suitable to capture capacity variations over cycle number [15]. The standard Wiener process, $X(\tau)$, can be expressed as follows [16]:

$$
X(\tau)=\mu \tau+\sigma B(\tau),
$$

where $\mu$ and $\sigma$ denote the drift and diffusion coefficient, respectively, and $\tau$ is time. The standard Brownian Motion has three properties: (1) $B(0)=0$; (2) $B(\tau+\Delta \tau)-B(\tau) \sim$ $N(0, \Delta \tau) ;(3) B(\tau) \sim N(0, \tau)$.

Apparently, (1) can only describe the linear degradation process over time. In engineering practice, a time transformation $\tau(t)=t^{\gamma}$ is often made to extend the standard Wiener process to the nonlinear applications [17]. Then, (1) can be rewritten as

$$
X(t)=\mu t^{\gamma}+\sigma B\left(t^{\gamma}\right) .
$$

The concept of first hitting time (FHT) is often used to define the failure time of $X(t)$ subject to a predefined critical value $\omega$, namely,

$$
T=\inf \{t \mid X(t) \geq \omega\} .
$$

In this paper, we use the time-transformation Wiener process $X(t)$ to describe the relationship between "capacity loss" and cycle number $t$. According to the engineering standard, the critical value $\omega$ can be set to be $30 \%$. Then, the probability density function (PDF) of battery cycle life can be derived as [17]

$$
\begin{aligned}
f_{T}(t) & =\frac{\partial F(t)}{\partial t} \\
& =\sqrt{\frac{\omega^{2}}{2 \pi \sigma^{2} t^{3 \gamma}}} \cdot \exp \left[-\frac{\left(\omega-\mu t^{\gamma}\right)^{2}}{2 \sigma^{2} t^{\gamma}}\right] \cdot r t^{\gamma-1} .
\end{aligned}
$$

To obtain the values of unknown model parameters, a maximum likelihood estimate (MLE) method is utilized. Let $x_{i, j}$ be the capacity loss of cell $i$ in cycle $j, i=1,2,3,4$ and $j=1,2, \cdots, n_{i} . n_{i}$ is the total cycle number of cell $i$. In this paper, $n_{1}=n_{2}=n_{3}=166$ and $n_{4}=131$. Then, let
$\Delta x_{i, j}=x_{i, j+1}-x_{i, j}$ denote the degradation increment between cycles $j$ and $j+1$. According to the properties of Wiener process, we have

$$
\Delta x_{i, j} \sim N\left(\mu \Delta \tau_{j}, \sigma^{2} \Delta \tau_{j}\right)
$$

where $\Delta \tau_{j}=(j+1)^{\gamma}-j^{\gamma}$.

The problem of parameter estimation can be transformed into maximizing the total log-likelihood function $\ln L\left(\mu, \sigma^{2}, \gamma\right)$, namely,

$$
\ln L\left(\mu, \sigma^{2}, \gamma\right)=\sum_{i=1}^{N} \sum_{j=1}^{M_{i}} \log \left(f\left(\Delta x_{i, j}\right)\right),
$$

where $f\left(\Delta x_{i, j}\right)=\left(1 / \sqrt{2 \pi \Delta \tau_{j}} \sigma\right) \exp \left(-\left(\Delta x_{i, j}-\mu \Delta \tau_{j}\right)^{2} /\right.$ $\left.2 \sigma^{2} \Delta \tau_{j}\right)$.

On the platform of Matlab 2017(a), the above optimization problem can be solved by the package "fminsearch." The estimators are $\widehat{\mu}=0.68, \widehat{\gamma}=0.75$, and $\widehat{\sigma}=1.60$.

2.3. Battery Offline Reliability Evaluation. Reliability evaluation is an essential task for satellite components with long lifetimes. The reliability of a certain type component can be expressed as a function of time, $R(t)$, which refers to their average normal working probability before $t$. Under the assumption of Wiener model, the reliability function of lithium-ion batteries in the test can be calculated by

$$
\begin{aligned}
R(t)= & P(T \leq t)=1-\int_{0}^{t} f_{T}(t) \mathrm{d} t \\
= & \Phi\left(\frac{\mu \cdot t^{r}-\omega}{\sigma \sqrt{t^{r}}}\right) \\
& +\exp \left(\frac{2 \mu \omega}{\sigma^{2}}\right) \Phi\left(\frac{-\mu \cdot t^{r}-\omega}{\sigma \sqrt{t^{r}}}\right) .
\end{aligned}
$$

Substituting the parameter estimators into (7), we can obtain the reliability $\widehat{R}(t)$ of this type of battery at any given time. In practice, many satellite designers are also concerned about the confidence intervals (CIs) of reliability indices, especially in the fields of risk evaluation and strategy optimization. Compared with a single point value, CIs contain more information on the description of statistical uncertainty. Motivated by [18], the reliability CIs of lithiumion batteries can be evaluated by the delta method. The variance of $\widehat{R}$ can be calculated approximately by

$$
\operatorname{var}(\widehat{R})=\boldsymbol{H} \cdot \boldsymbol{I}^{-\mathbf{1}} \cdot \boldsymbol{H}^{\prime},
$$

where

$$
\begin{aligned}
& \boldsymbol{H}=\left[\frac{\partial R(t)}{\partial \mu}, \frac{\partial R(t)}{\partial \sigma}, \frac{\partial R(t)}{\partial \gamma}\right],
\end{aligned}
$$

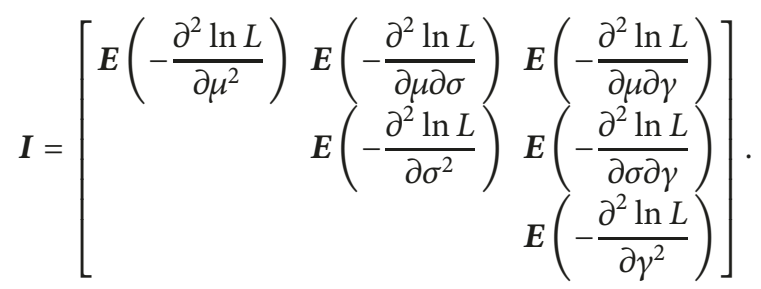


The elements in vector $\boldsymbol{H}$ and the fisher information matrix $\boldsymbol{I}$ are given as follows:

$$
\begin{gathered}
\frac{\partial R(t)}{\partial \mu}=-\frac{\sqrt{t^{\gamma}}}{\sigma} \cdot \phi\left(\frac{\mu \cdot t^{\gamma}-\omega}{\sigma \sqrt{t^{\gamma}}}\right)-\frac{2 \omega}{\sigma^{2}} \cdot \exp \left(\frac{2 \mu \omega}{\sigma^{2}}\right) \\
\cdot \Phi\left(\frac{-\mu \cdot t^{\gamma}-\omega}{\sigma \sqrt{t^{\gamma}}}\right)+\frac{\sqrt{t^{\gamma}}}{\sigma} \cdot \exp \left(\frac{2 \mu \omega}{\sigma^{2}}\right) \\
\cdot \phi\left(\frac{-\mu \cdot t^{\gamma}-\omega}{\sigma \sqrt{t^{\gamma}}}\right) \\
\frac{\partial R(t)}{\partial \sigma}=\frac{\mu \cdot t^{\gamma}-\omega}{\sigma^{2} \sqrt{t^{\gamma}}} \cdot \phi\left(\frac{\mu \cdot t^{\gamma}-\omega}{\sigma \sqrt{t^{\gamma}}}\right)+\frac{4 \mu \omega}{\sigma^{3}} \\
\cdot \exp \left(\frac{2 \mu \omega}{\sigma^{2}}\right) \cdot \Phi\left(\frac{-\mu \cdot t^{\gamma}-\omega}{\sigma \sqrt{t^{\gamma}}}\right)+\frac{-\mu \cdot t^{\gamma}-\omega}{\sigma^{2} \sqrt{t^{\gamma}}} \\
\cdot \exp \left(\frac{2 \mu \omega}{\sigma^{2}}\right) \cdot \phi\left(\frac{-\mu \cdot t^{\gamma}-\omega}{\sigma \sqrt{t^{\gamma}}}\right) \cdot \\
\frac{\partial R(t)}{\partial \gamma}=-\phi\left(\frac{\mu \cdot t^{\gamma}-\omega}{\sigma \sqrt{t^{\gamma}}}\right) \frac{\mu t^{\gamma / 2}-\omega t^{-\gamma / 2}}{2 \sigma} \ln \sqrt{t} \\
-\exp \left(\frac{2 \mu \omega}{\sigma^{2}}\right) \phi\left(\frac{-\mu \cdot t^{\gamma}-\omega}{\sigma \sqrt{t^{\gamma}}}\right) \frac{-\mu t^{\gamma / 2}-\omega t^{-\gamma / 2}}{2 \sigma}
\end{gathered}
$$$$
\cdot \ln \sqrt{t}
$$$$
\boldsymbol{E}\left(-\frac{\partial^{2} \ln L}{\partial \mu^{2}}\right)=\frac{1}{\sigma^{2}} \sum_{i=1}^{N} \sum_{j=1}^{M_{i}}\left(t_{j}^{\gamma}-t_{j-1}^{\gamma}\right)
$$$$
\boldsymbol{E}\left(-\frac{\partial^{2} \ln L}{\partial \mu \partial \sigma}\right)=0
$$$$
\boldsymbol{E}\left(-\frac{\partial^{2} \ln L}{\partial \mu \partial \gamma}\right)=\frac{\mu}{\sigma^{2}} \sum_{i=1}^{N} \sum_{j=1}^{M_{i}}\left(t_{j}^{\gamma} \ln t_{j}-t_{j-1}^{\gamma} \ln t_{j-1}\right)
$$$$
\boldsymbol{E}\left(-\frac{\partial^{2} \ln L}{\partial \sigma^{2}}\right)=\frac{2}{\sigma^{2}} \sum_{i=1}^{N} M_{i}
$$$$
\boldsymbol{E}\left(-\frac{\partial^{2} \ln L}{\partial \sigma \partial \gamma}\right)=\frac{1}{\sigma} \sum_{i=1}^{N} \sum_{j=1}^{M_{i}} \frac{t_{j}^{\gamma} \ln t_{j}-t_{j-1}^{\gamma} \ln t_{j-1}}{t_{j}^{\gamma}-t_{j-1}^{\gamma}}
$$$$
\boldsymbol{E}\left(-\frac{\partial^{2} \ln L}{\partial \gamma^{2}}\right)=\frac{1}{2 \sigma^{2}}
$$$$
\cdot \sum_{i=1}^{N} \sum_{j=1}^{M_{i}}\left[\frac{2 \mu^{2}\left[\left(t_{j}^{\gamma} \ln t_{j}-t_{j-1}^{\gamma} \ln t_{j-1}\right)^{2}\right]}{\left(t_{j}^{\gamma}-t_{j-1}^{\gamma}\right)}\right.
$$$$
\left.+\frac{\sigma^{2}\left[\left(t_{j}^{\gamma} \ln t_{j}-t_{j-1}^{\gamma} \ln t_{j-1}\right)^{2}\right]}{\left(t_{j}^{\gamma}-t_{j-1}^{\gamma}\right)^{2}}\right] .
$$

Then, it is reasonable to assume that the reliability function $R(t)$ approximately follows the normal distribution
TABLE 1: Offline life perdition results of satellite lithium-ion batteries (cycles).

\begin{tabular}{lcc}
\hline Name & Point estimate & $85 \%$ CIs \\
\hline Mean life & 160 & {$[143,178]$} \\
Median life & 145 & {$[130,162]$} \\
60 percentile life & 128 & {$[115,143]$} \\
70 percentile life & 114 & {$[102,126]$} \\
80 percentile life & 98 & {$[88,109]$} \\
90 percentile life & 80 & {$[72,89]$} \\
\hline
\end{tabular}

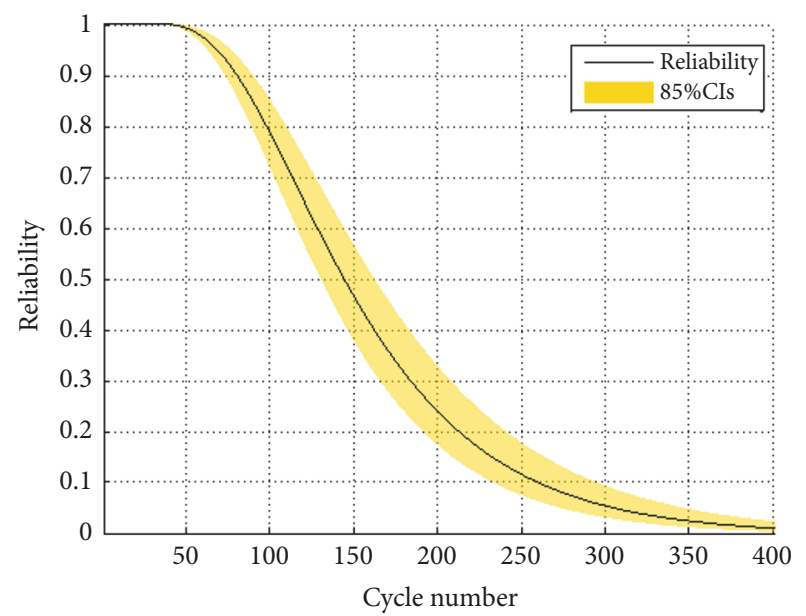

FIGURE 5: Reliability evaluation results of satellite lithium-ion batteries in NASA test.

$N(\widehat{R}(t), \operatorname{var}(\widehat{R}))$. Thus, the $100(1-\alpha) \%$ CIs of $R(t)$ can be calculated by

$$
\left[\widehat{R}-z_{1-\alpha / 2} \cdot \sqrt{\operatorname{var}(\widehat{R})}, \widehat{R}+z_{1-\alpha / 2} \cdot \sqrt{\operatorname{var}(\widehat{R})}\right],
$$

where $z_{1-\alpha / 2}$ is the inverse of the standard normal distribution corresponding to quantile $1-\alpha / 2$.

With the above methods, the point evaluation and $85 \%$ CIs of the reliability curve of satellite lithium-ion batteries in NASA test are presented in Figure 5. Besides reliability curves, some indices of interests are needed in satellite design and management. Table 1 gives the predictions of several typical indices defined by (9) and (10). It is noted that the 50 percentile life is also called the median life.

$$
\begin{aligned}
\text { Mean life } & =\int_{0}^{\infty} t \cdot f_{T}(t) \mathrm{d} t, \\
q \text { percentile life } & =\{t \mid R(t)=q \%\} .
\end{aligned}
$$

\section{Battery In-Orbit Capacity Prediction}

3.1. In-Orbit Charge/Discharge Prolife Analysis. In this study, acquiring the "capacity loss" in each cycle is the prerequisite of performing offline prediction. As mentioned above, battery capacity can be measured accurately through the Amperehour integral method, which needs to conduct a complete charge/discharge process. However, the charge/discharge 
TABLE 2: Availability status of batteries parameters in orbit environments.

\begin{tabular}{lccc}
\hline Phase & Parameter & Status & Reason \\
\hline CC charge & Voltage & partially available & starting point is random \\
CV charge & Current & all available & sensitive to discharging current \\
CC discharge & Voltage & unavailable & .
\end{tabular}

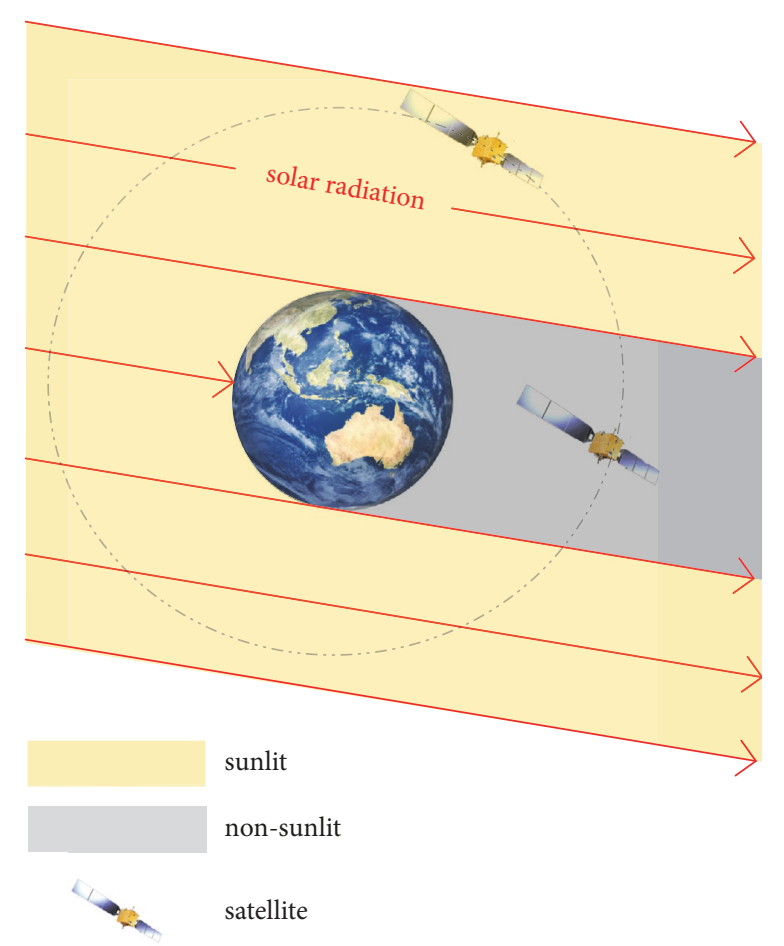

FIGURE 6: Diagram of sunlit and non-sunlit periods for an in-orbit satellite.

profiles of a battery in orbits are quite different from that under laboratory conditions. As we know, a satellite rotates around Earth, and Earth revolves around the sun. Consequently, a satellite in orbit periodically experiences sunlit period and non-sunlit period, as Figure 6 shows. In the sunlit period, the solar panels on the satellite expand to absorb solar radiation and produce electricity, which is used to power satellite loadings and charge batteries (also in CC-CV regime). In the non-sunlit period, as the solar radiation is blocked by Earth, satellite loadings completely rely on the secondary batteries for power supply. Thus, the charge/discharge profiles in orbit differ from those in laboratory (as Figure 3 shows) in two respects: (1) In CC discharging, both current and depth-of-discharge (DOD) are determined by satellite loadings and always vary in different cycles; (2) in CC charging, the starting point of voltage curve in cycle $j$ is determined by the DOD in cycle $(j-1)$. Unlike the $\mathrm{CC}$ charing, the $\mathrm{CV}$ charging process in orbit and laboratory stays the same.

The availability status of battery parameters in orbit is summarized in Table 2. Due to the lack of full current curves in both charge and discharge, battery capacity cannot be calculated directly through the time integration of current. Fortunately, the partial charging data also contain abundant information on battery health status. In the next subsection, an empirical model for capacity prediction is studied with the incomplete charging data.

3.2. In-Orbit Capacity Prediction Modeling. To ensure reliability and safety, the DOD of most satellite batteries in one cycle is designed to be less than $40 \%$. As a result, although the starting point of CC charging varies in different cycles, most of them are located in the linear part of the voltage curve. In Figure 7(a), the circles denote the starting charging points in each cycle. Thus, for in-orbit satellite batteries, the relationship between voltage and time in CC charging can be modeled by a linear function:

$$
V(t)=a \times t+b .
$$

Unlike the voltage, the complete current curve in $\mathrm{CV}$ charging can be monitored. In a specified cycle, the charging voltage declines over time with a decreasing rate. By analyzing the dataset of NASA, we find that a power function is quite suitable to describe this rule, namely,

$$
I(t)=c \times t^{d}
$$

With cycle number accumulating, a lithium-ion battery degrades and the voltage and current curves in charging also change accordingly. Figure 8 depicts the variations of parameters $a, b, c, d$ over battery capacity, respectively. Except $a$, the other three parameters correlate strongly with battery capacity. Thus, it is natural to attempt to build a capacity prediction model considering the above correlations.

Due to lack of prior knowledge on the forms of capacity prediction model, a general tri-variate regression model is adopted, namely,

$$
\begin{aligned}
\operatorname{Cap}(b, c, d)= & x_{1} b+x_{2} c+x_{3} d+x_{4} b^{2}+x_{5} c^{2}+x_{6} d^{2} \\
& +x_{7} b c+x_{8} b d+x_{9} c d+x_{10} .
\end{aligned}
$$

where $x_{1}, x_{2}, \cdots, x_{10}$ are the regression coefficients, which can be estimated by the least square method.

However, a common problem of multiple regression is that not all of the variables are significant and necessary. Generally, statisticians utilize the stepwise regression method to select effective predictive variables. In each step, a variable is considered for addition (forward selection) or subtraction (backward elimination) from the set of explanatory variables based on F-tests or t-tests [19]. In this paper, the backward elimination algorithm is used to perform stepwise regression for (10), and the results are shown in Figure 9 (supplied by 


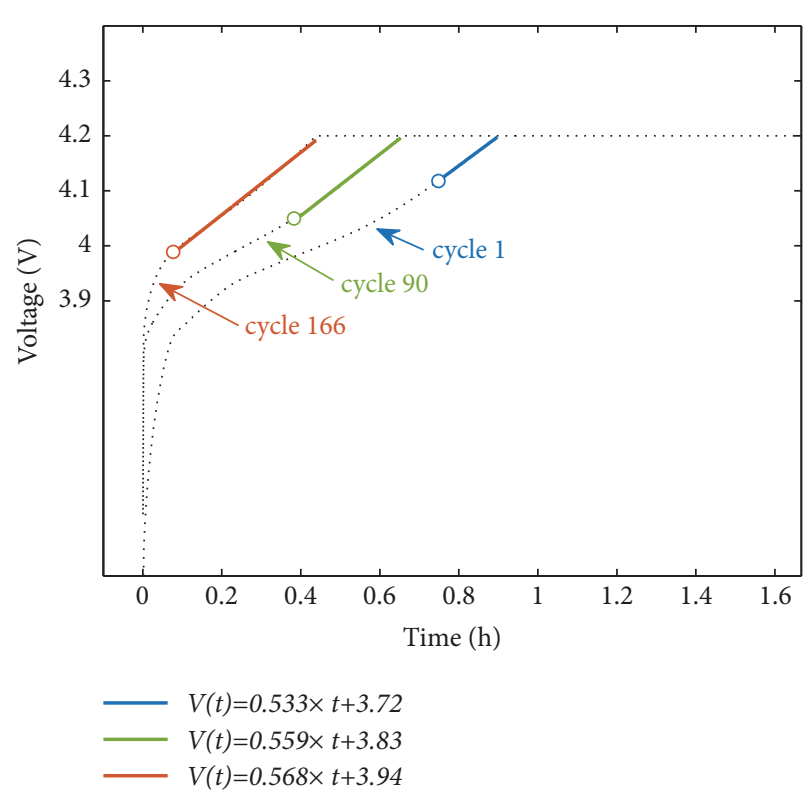

(a) Voltage

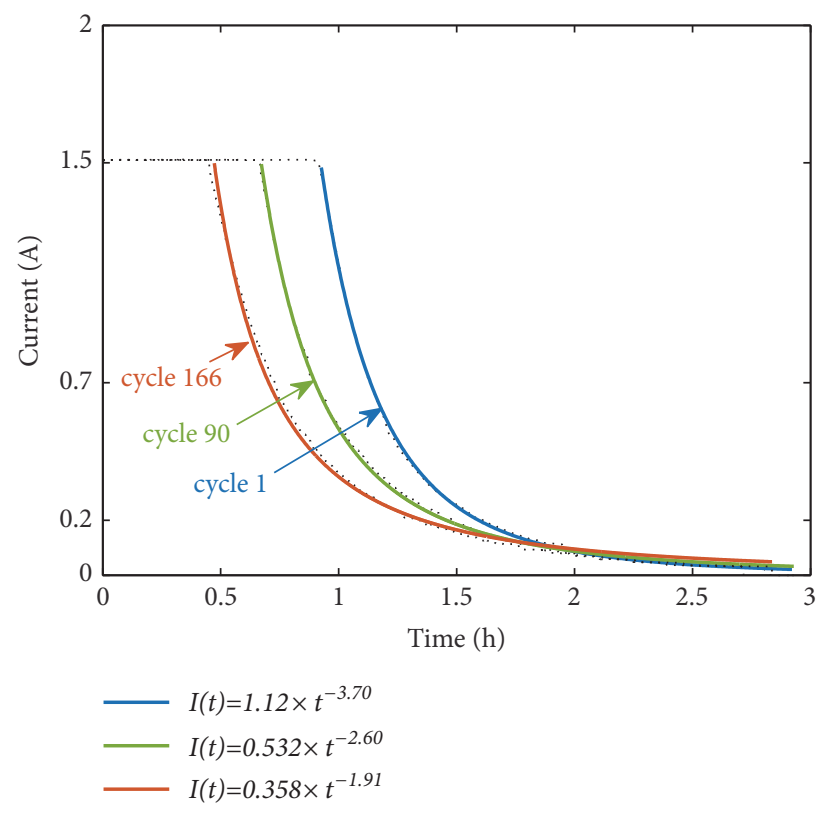

(b) Current

FIGURE 7: Voltage and current fitting in CC-CV charging.
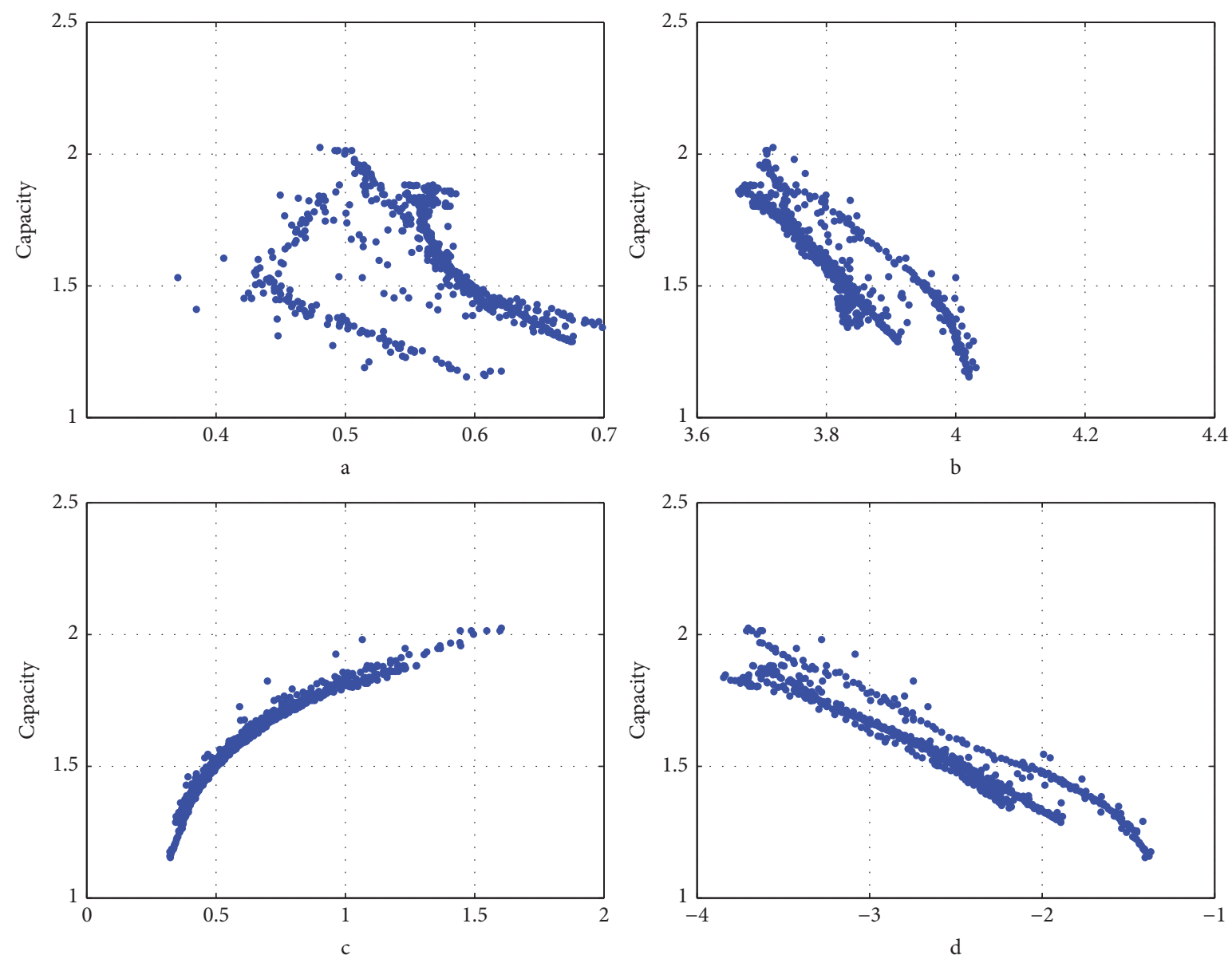

FIGURE 8: Correlation analysis results. 

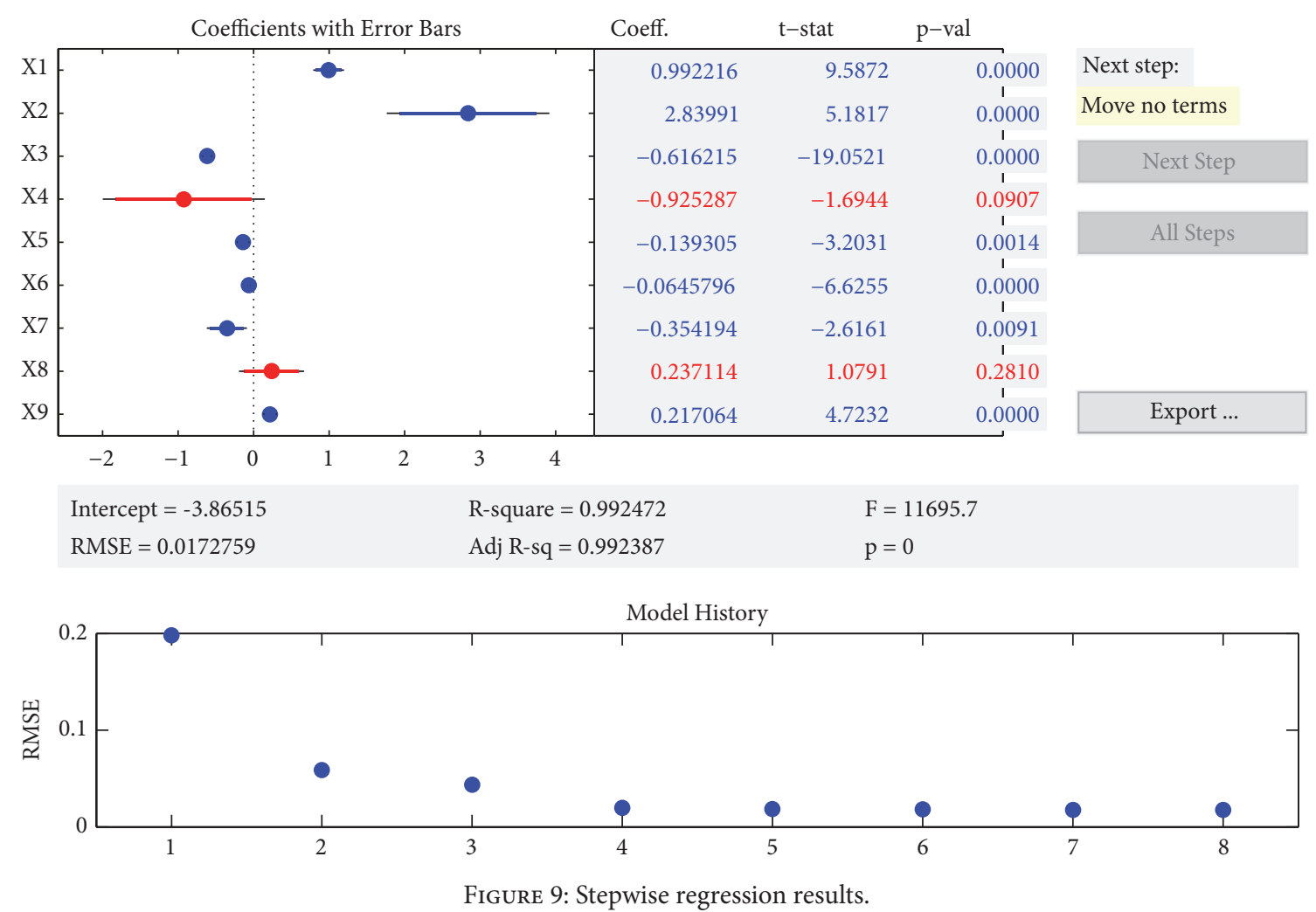

the "stepwise" package in Matlab 2017(a)). The least RMSE corresponds to the optimal result in the stepwise regression and the blue points refer to the remaining variables. It can be found that the variables $b^{2}$ and $b d$ are invalid and should be removed. Then, the capacity prediction model reduces to

$$
\begin{aligned}
\operatorname{Cap}(b, c, d)= & x_{1} b+x_{2} c+x_{3} d+x_{5} c^{2}+x_{6} d^{2}+x_{7} b c \\
& +x_{9} c d+x_{10}
\end{aligned}
$$

where $x_{1}=0.91, x_{2}=2.23 x_{3}=-0.58, x_{5}=-0.075, x_{6}=$ $-0.051, x_{7}=-0.20, x_{9}=0.25$, and $x_{10}=-3.51$.

3.3. Model Validation. The coefficients in (17) are estimated using the dataset of all the 4 batteries in the NASA test. To further demonstrate the model's prediction ability, the leave-one-out cross-validation (LOOCV) is conducted. In the LOOCV, we each time leave one battery out to perform capacity prediction by (17) and use the other three batteries' data to estimate regression function coefficients. The curves of measured and predicted capacity of the four batteries are given in Figure 10, respectively.

In most cycles, the predicted capacity matches quite well with the measured value. Results indicate that the proposed model not only has high fitting accuracy, but also has strong forecasting ability. However, it should be noted that the prediction results of B0005 and B0006 in several early cycles (less than 15) are not accurate enough. This phenomenon may be explained by the internal discrepancy among different battery samples.

\section{In-Orbit Residual Life Prediction}

With the predicted capacity in each cycle, the in-orbit life prediction for satellite lithium-ion batteries can be realized. Unlike the offline life prediction, which focuses on the battery population's reliability evaluation, in-orbit prediction concerns more on the residual life of each individual battery. Reliability evaluation only reflects the average level of lifetime for a specified type of product. However, the degradation processes of individual items show some heterogeneity due to randomness in materials, manufactures, or environments [20]. As Figure 11 shows, the fitted degradation path of different units varies drastically, resulting in a significant difference in EOL. Thus, the key technique of RL prediction is to update each individual battery's degradation model timely with the accumulation of capacity fading measurement.

Under the Bayesian framework, the parameters in a degradation model can be divided into fixed effects and random effects. The former is assumed to be constant to capture the common degradation characteristics in a population, and the latter is randomly distributed to capture the item-totime variability. For the capacity fading model in (2), the drift parameter $\mu$ determines degradation rate and thus is always assumed to be the random effect which follows a normal distribution; namely, $\pi(\mu) \sim N\left(\alpha_{0}, \beta_{0}^{2}\right)$. The time transformation parameter $\gamma$ and diffusion parameter $\sigma$, which determine the degradation path's shape and uncertainty, are always assumed to be fixed effects [21]. The hyperparameters $\alpha_{0}$ and $\beta_{0}$ can be estimated by the MLE method with the 

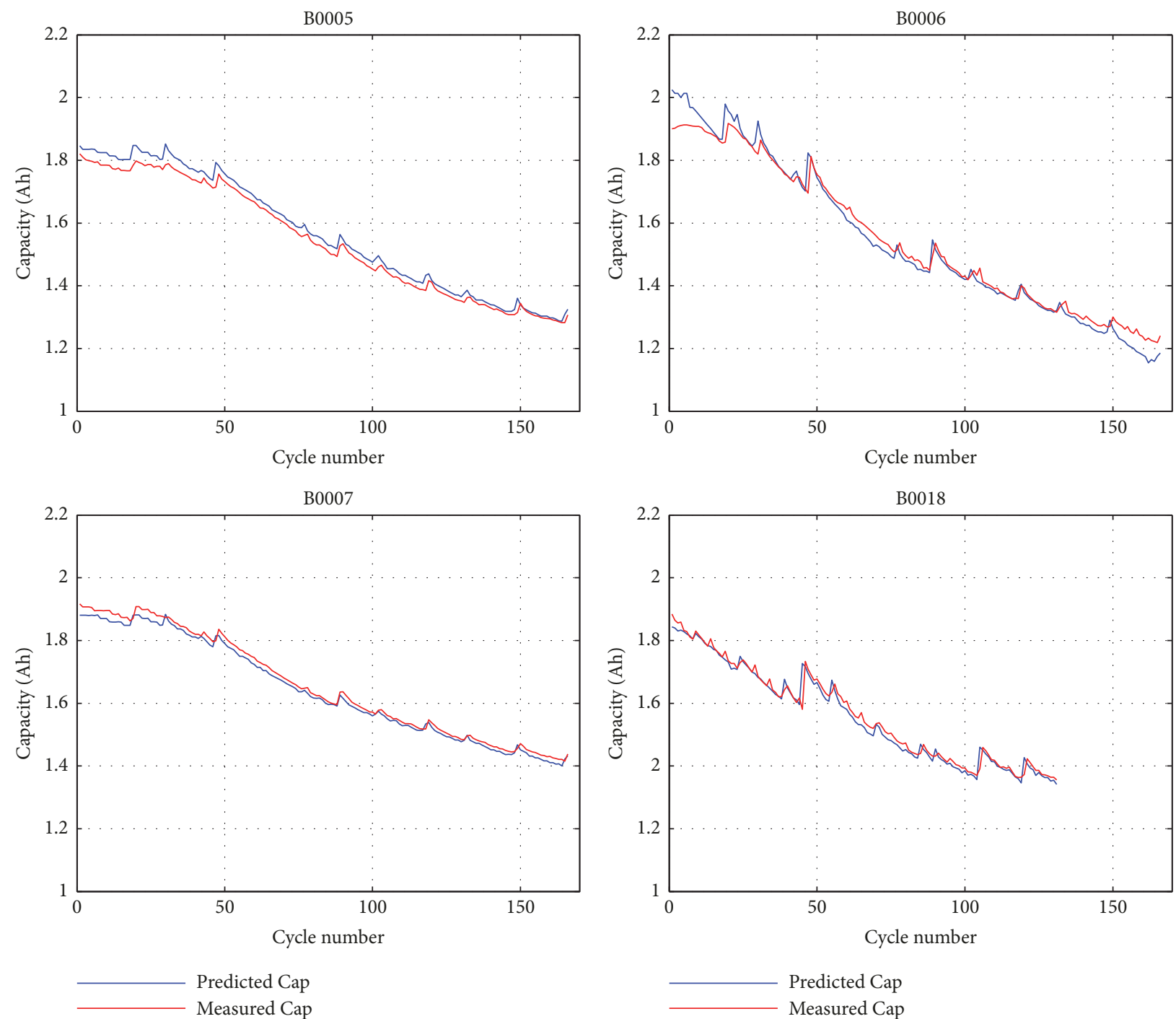

FIgURE 10: Capacity prediction results in LOOCV.

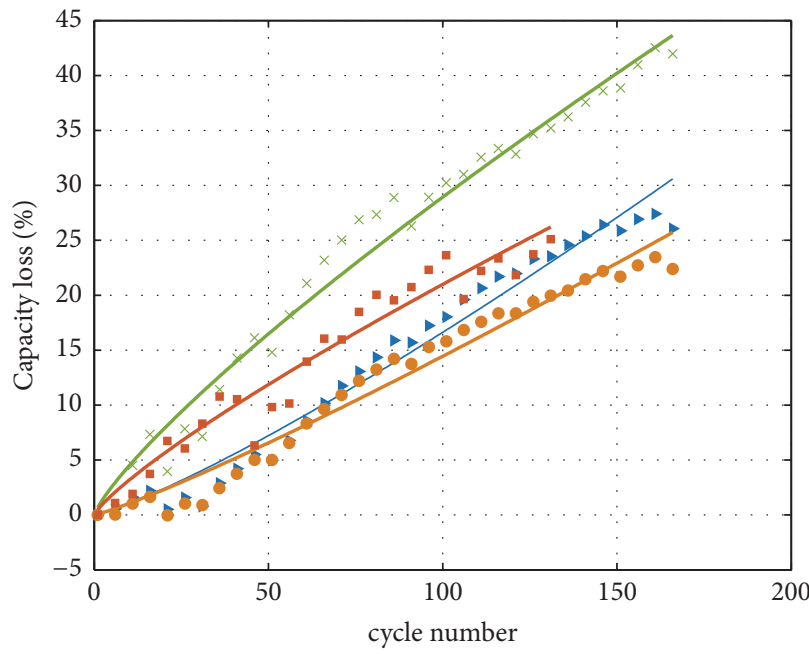

FIGURE 11: Fitting results of $\mu t^{\gamma}$ for each battery. 
four estimators of drift parameters considering each battery's capacity loss individually. The results are $\alpha_{0}=0.67, \beta_{0}=0.19$.

A premise of RL prediction is to ensure that the object battery's capacity fading model approximates the actual degradation path, which can be realized by the Bayesian method. Let $x_{1}, x_{2}, \cdots, x_{k}$ be the "capacity loss" in cycle $1,2, \cdots, k$. For an in-orbit battery, once new "capacity loss" is available, the posterior distribution of $\mu$ at cycle $k$ can be updated as follows:

$$
\pi\left(\mu \mid x_{1: k}\right) \propto f\left(x_{1: k} \mid \mu\right) \pi(\mu)
$$

where $f\left(x_{1: k} \mid \mu\right)$ is the likelihood of degradation increment,

$$
\begin{aligned}
& f\left(x_{1: k} \mid \mu\right) \\
& =\prod_{i=1}^{k} \frac{1}{\sqrt{2 \pi \sigma^{2} \Delta \tau_{i}}} \exp \left\{\frac{-\left(\Delta x_{i}-\mu \Delta \tau_{i}\right)^{2}}{2 \sigma^{2} \Delta \tau_{i}}\right\}, \\
& \pi(a)=\frac{1}{\sqrt{2 \pi \beta^{2}}} \exp \left\{\frac{-(a-\alpha)^{2}}{2 \beta^{2}}\right\} .
\end{aligned}
$$

It is easy to prove that the posterior distribution $\pi(\mu$ । $x_{1: k}$ ) also follows a normal distribution with mean $\mu_{k}$ and variance $\sigma_{k}^{2}$, where

$$
\begin{aligned}
\mu_{k} & =\frac{\beta^{2} \sum_{i=1}^{k} \Delta x_{i}+\alpha \sigma^{2}}{\sigma^{2}+\beta^{2} \sum_{i=1}^{k} \Delta \tau_{i}} \\
\sigma_{k} & =\frac{\beta^{2} \sigma^{2}}{\sigma^{2}+\beta^{2} \sum_{i=1}^{k} \Delta \tau_{i}}
\end{aligned}
$$

RL measures the length of time an in-orbit battery can work form the current cycle. At cycle $k$, the residual life $R L_{k}$ is defined as [22]

$$
R L_{k}=\inf \left\{t \mid X(t) \geq \omega-x_{k}\right\}
$$

Motivated by [23], the PDF of $R L_{k}$ can be calculated as follows:

$$
f_{k}(t)=\frac{g(t)}{\int_{0}^{\infty} g(t) \mathrm{d} t}
$$

where

$$
\begin{aligned}
& g(t)=\frac{\omega-x_{k}}{\Delta \tau(k+t) \sqrt{2 \pi U_{k}}} \\
& \quad \cdot \exp \left(-\frac{\left[\omega-x_{k}-\mu_{k} \Delta \tau(k+t)\right]^{2}}{2 U_{k}}\right) \gamma(k+t)^{\gamma-1,} \\
& \Delta \tau(k+t)=(k+t)^{\gamma}-k^{\gamma}, \\
& U_{k}=\Delta \tau(k+t)\left(\sigma_{k}^{2}+\sigma^{2}\right) .
\end{aligned}
$$

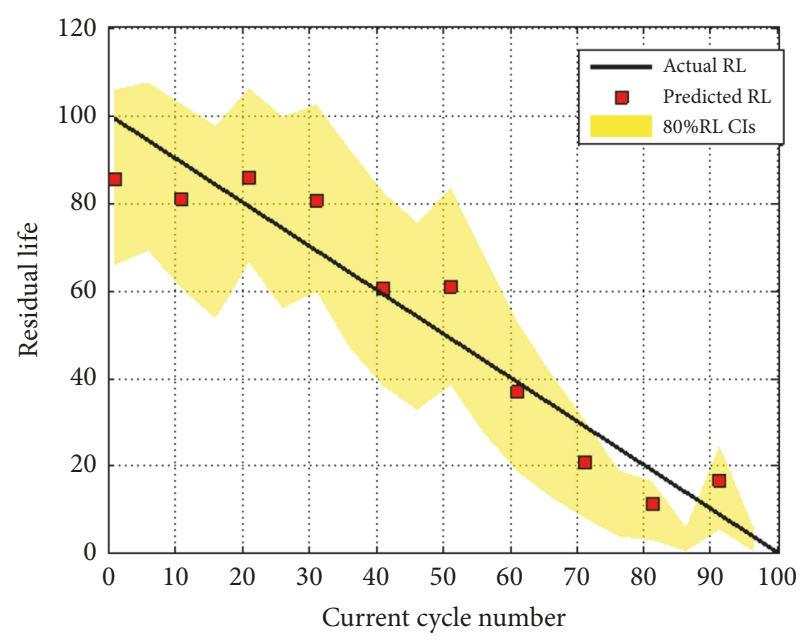

FIGURE 12: Residual life predictions of B0006.

Then, we can calculate the mean and $100(1-\alpha) \%$ CIs of RL to perform in-orbit lithium-ion battery RL prediction; namely,

$$
\begin{aligned}
\text { mean } R L_{k} & =\int_{0}^{\infty} t \cdot f_{k}(t) \mathrm{d} t \\
\int_{0}^{R L_{k}^{L}} f_{k}(t) \mathrm{d} t & =\frac{\alpha}{2} \\
\int_{0}^{R L_{k}^{U}} f_{k}(t) \mathrm{d} t & =1-\frac{\alpha}{2},
\end{aligned}
$$

where the superscripts "L" and " $U$ " represent the confidence lower and upper limit, respectively.

According to the failure criteria, only B0006's "capacity loss" reaches the threshold $\omega=30 \%$. To illustrate the proposed method and demonstrate its effectiveness, B0006's data are utilized to perform RL prediction. The results are shown in Figure 12. The drift parameter is updated every 10 cycles and the mean (red square) and $80 \%$ CIs of RL are calculated and plotted sequentially. Since the Bayesian updating procedure ensures the accuracy of B0006's capacity fading model, the actual RLs of B0006 fall within the ranges of $80 \%$ CIs and are close to the mean predictions in most cycles.

\section{Conclusions}

Lithium-ion battery is the core component of modern satellite power subsystem. Engineers often use useable capacity to measure a battery's degradation and aging. In this paper, a framework of satellite lithium-ion battery life prediction is developed, which is suitable for both the online and offline situations. In the offline stage, battery capacity is measured by the classic Ampere-hour integral method with the current data. A Wiener process with time transformation is used to model capacity fading over cycle number and to infer battery reliability function. The delta method is used to calculate the confidence intervals of reliability function and life indices. 
In the online stage, we firstly propose an empirical regression model to realize accurate capacity prediction with the incomplete charge data, and then use the Bayesian method to perform capacity fading model updating and battery residual life prediction. The dataset of NASA lithium-ion battery test is used to validate these methods. Results show that the accuracy of both capacity prediction and RL prediction reaches an acceptable level, and the proposed approaches can be easily extended to other lithium-ion battery applications (like the EV) and even other types of secondary batteries.

Some limitations of this study lie in the following: (1) the sample size in NASA test is small; (2) there is no consideration of the effects of environmental stress like temperature and discharging current. In the future, we will further refine and validate the prediction model with more battery degradation data, from both additional ground tests and in-orbit telemetry.

\section{Data Availability}

The data used to support the findings of this study are available from the corresponding author upon request.

\section{Conflicts of Interest}

The authors declare that they have no conflicts of interest.

\section{Acknowledgments}

The authors would like to acknowledge NASA for the lithiumion battery testing data, which are publicly available over the web for download.

\section{References}

[1] X. Xi, W. Xu, X. Bai et al., "A smart remaining battery life prediction based on MARS," in Proceedings of the 2014 IEEE Power \& Energy Society Innovative Smart Grid Technologies Conference (ISGT), pp. 1-5, Washington, DC, USA, Feburary 2014.

[2] S. J. Tang, C. Q. Yu, X. Wang, X. S. Guo, and X. S. Si, "Remaining useful life prediction of lithium-ion batteries based on the Wiener process with measurement error," Energies, vol. 7, no. 2, pp. 520-547, 2014.

[3] K. Goebel, B. Saha, A. Saxena, J. R. Celaya, and J. P. Christophersen, "Prognostics in battery health management," IEEE Instrumentation \& Measurement Magazine, vol. 11, no. 4, pp. 3340, 2008.

[4] N. Williard, W. He, C. Hendricks, and M. Pecht, "Lessons learned from the 787 dreamliner issue on Lithium-Ion Battery reliability," Energies, vol. 6, no. 9, pp. 4682-4695, 2013.

[5] H. P. Wu and Y. H. Luan, "An Efficient Estimation of the Mean Residual Life Function with Length-Biased Right-Censored Data," Mathematical Problems in Engineering, vol. 2014, Article ID 937397, 5 pages, 2014.

[6] Y. Liu, B. He, F. Liu, S. Lu, Y. Zhao, and J. Zhao, "Remaining Useful Life Prediction of Rolling Bearings Using PSR, JADE, and Extreme Learning Machine," Mathematical Problems in Engineering, vol. 2016, Article ID 8623530, 13 pages, 2016.
[7] G. Jin, D. E. Matthews, and Z. Zhou, "A Bayesian framework for on-line degradation assessment and residual life prediction of secondary batteries in spacecraft," Reliability Engineering \& System Safety, vol. 113, no. 1, pp. 7-20, 2013.

[8] Y. Xing, E. W. M. Ma, K. Tsui, and M. Pecht, "An ensemble model for predicting the remaining useful performance of lithium-ion batteries," Microelectronics Reliability, vol. 53, no. 6, pp. 811-820, 2013.

[9] B. Saha and K. Goebel, "Modeling li-ion battery capacity depletion in a particle filtering framework," in Proceedings of the Annual Conference of the Prognostics and Health Management Society, p. 10, San Diego, CA, USA, 2009.

[10] A. Nuhic, T. Terzimehic, T. Soczka-Guth, M. Buchholz, and K. Dietmayer, "Health diagnosis and remaining useful life prognostics of lithium-ion batteries using data-driven methods," Journal of Power Sources, vol. 239, no. 1, pp. 680-688, 2013.

[11] D. H. Liu, H. Wang, Y. Peng, W. Xie, and H. T. Liao, "Satellite lithium-ion battery remaining cycle life prediction with novel indirect health indicator extraction," Energies, vol. 6, no. 8, pp. 3654-3668, 2013.

[12] B. Saha, K. Goebel, S. Poll, and J. Christophersen, "Prognostics methods for battery health monitoring using a Bayesian framework," IEEE Transactions on Instrumentation and Measurement, vol. 58, no. 2, pp. 291-296, 2009.

[13] J. Feng, P. Kvam, and Y. Tang, "Remaining useful lifetime prediction based on the damage-marker bivariate degradation model: A case study on lithium-ion batteries used in electric vehicles," Engineering Failure Analysis, vol. 70, pp. 323-342, 2016.

[14] “Data "B. Saha and K. Goebel (2007). ”Battery Data Set”, NASA Ames Prognostics Data Repository," [http://ti.arc.nasa.gov/ project/prognostic-data-repository], NASA Ames, Moffett Field, CA, USA.

[15] T. Liu, Q. Sun, J. Feng, Z. Pan, and Q. Huangpeng, "Residual life estimation under time-varying conditions based on a Wiener process," Journal of Statistical Computation and Simulation, vol. 87, no. 2, pp. 211-226, 2017.

[16] H. Li, D. Pan, and C. L. P. Chen, "Reliability modeling and life estimation using an expectation maximization based wiener degradation model for momentum wheels," IEEE Transactions on Cybernetics, vol. 45, no. 5, pp. 955-963, 2015.

[17] G. A. Whitmore and F. Schenkelberg, "Modelling accelerated degradation data using wiener diffusion with a time scale transformation," Lifetime Data Analysis, vol. 3, no. 1, pp. 27-45, 1997.

[18] T. Liu, Q. Sun, J. Meng, Z. Pan, and Y. Tang, "Degradation modeling of satellite thermal control coatings in a low earth orbit environment," Solar Energy, vol. 139, pp. 467-474, 2016.

[19] J. Mark and M. A. Goldberg, "Multiple regression analysis and mass assessment: a review of the issues," The Appraisal Journal, pp. 89-109, 2001.

[20] W. Wang, M. Carr, W. Xu, and K. Kobbacy, "A model for residual life prediction based on Brownian motion with an adaptive drift," Microelectronics Reliability, vol. 51, no. 2, pp. 285-293, 2011.

[21] X. S. Si, W. Wang, C. H. Hu, D. H. Zhou, and M. G. Pecht, "Remaining useful life estimation based on a nonlinear diffusion degradation process," IEEE Transactions on Reliability, vol. 61, no. 1, pp. 50-67, 2012.

[22] X. S. Si, W. Wang, M. Y. Chen, C. H. Hu, and D. H. Zhou, "A degradation path-dependent approach for remaining useful life 
estimation with an exact and closed-form solution," European Journal of Operational Research, vol. 226, no. 1, pp. 53-66, 2013.

[23] X. Wang, N. Balakrishnan, and B. Guo, "Residual life estimation based on a generalized Wiener degradation process," Reliability Engineering \& System Safety, vol. 124, pp. 13-23, 2014. 


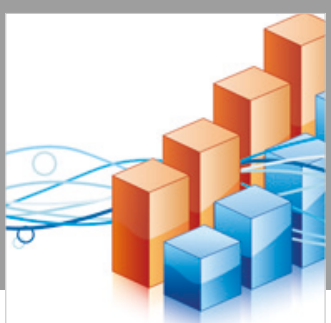

Advances in

Operations Research

\section{-n-m}
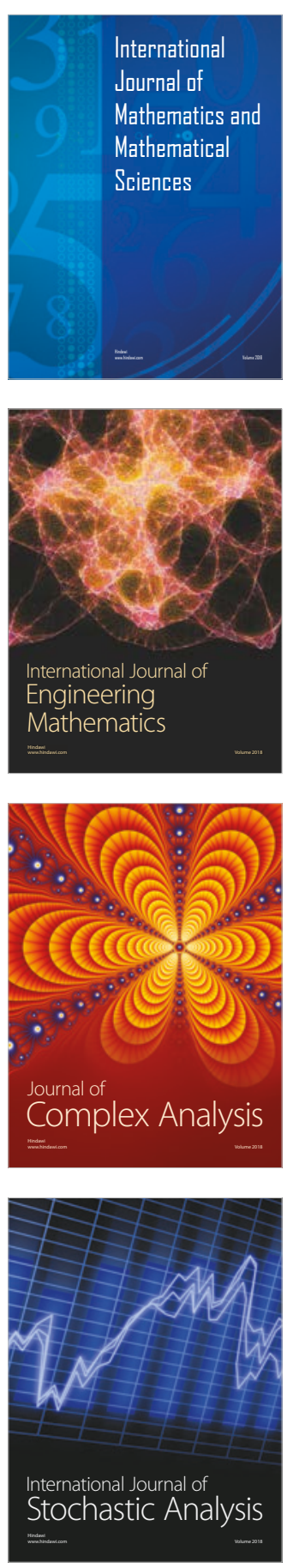
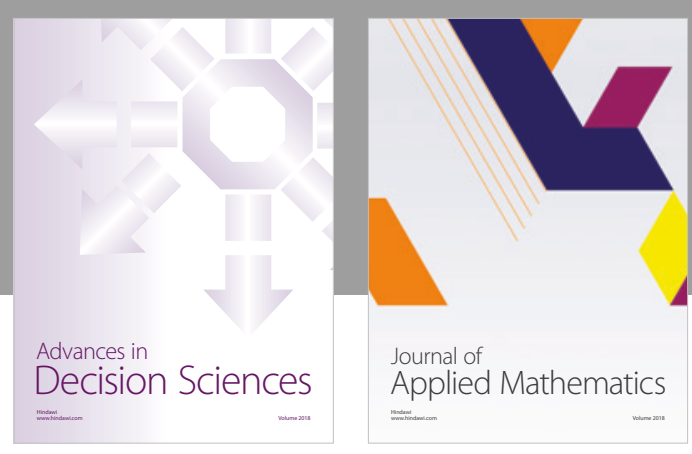

Journal of

Applied Mathematics
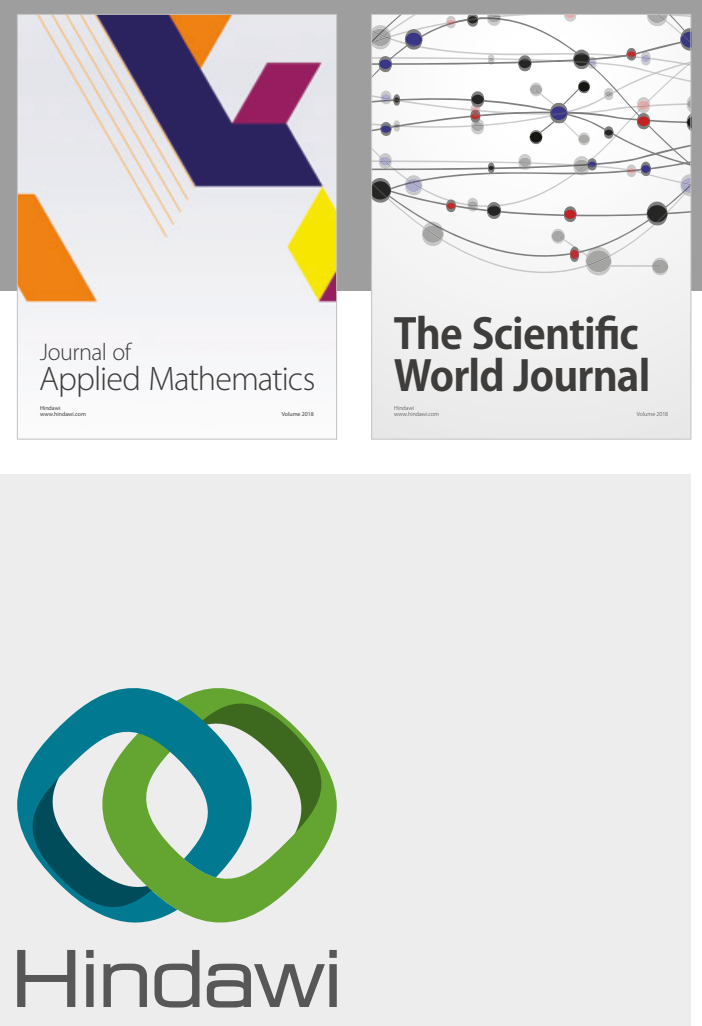

Submit your manuscripts at

www.hindawi.com

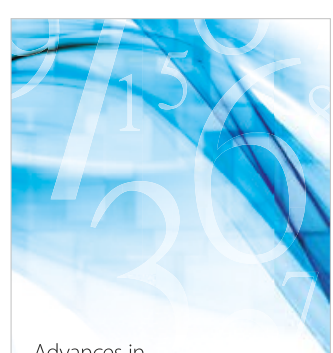

Advances in
Numerical Analysis
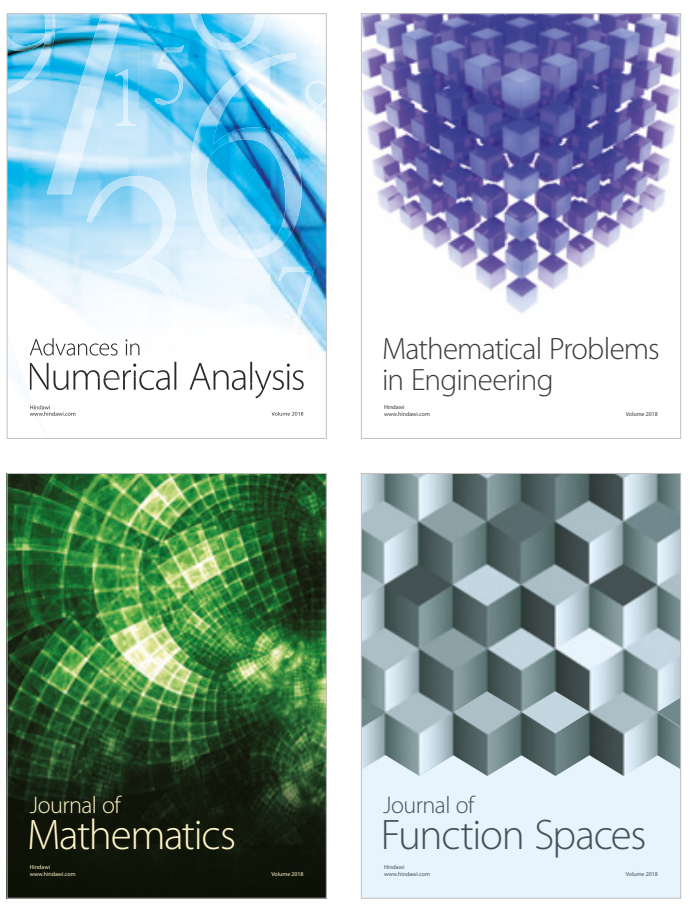

Mathematical Problems in Engineering

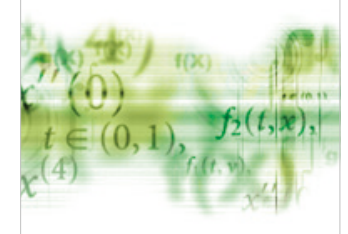

International Journal of

Differential Equations

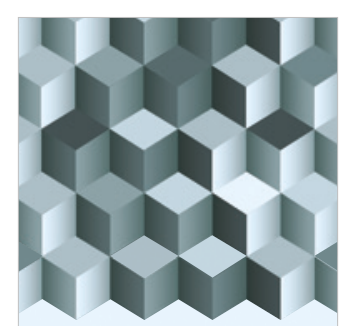

Journal of

Function Spaces

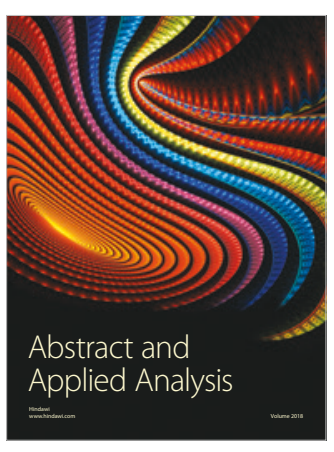

The Scientific

World Journal

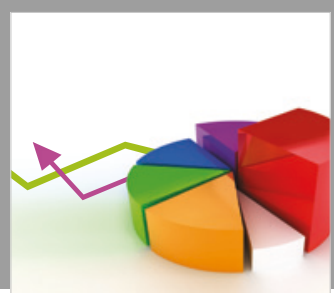

Journal of

Probability and Statistics
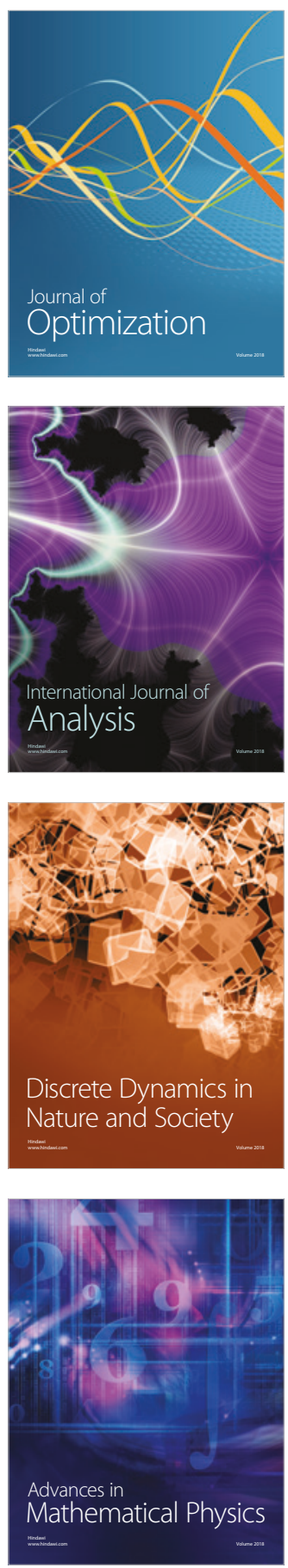